\title{
Platelet-derived growth factor- $\beta$ expression in rabbit models of cerebral vasospasm following subarachnoid hemorrhage
}

\author{
HONG-KAI CUI ${ }^{1}$, RUI-FANG YAN ${ }^{2}$, XIAO-LI DING ${ }^{3}$, PENG ZHAO $^{1}$, QING-WU WU ${ }^{2}$, \\ HONG-PO WANG ${ }^{2}$, HAI-XIA QIN ${ }^{3}$, JIAN-FEI TU ${ }^{4}$ and RUI-MIN YANG ${ }^{1}$ \\ Departments of ${ }^{1}$ Interventional Radiology, ${ }^{2}$ Radiology and ${ }^{3}$ Obstetrics and Gynecology, \\ The First Affiliated Hospital of Xinxiang Medical University, Weihui, Henan 453100; \\ ${ }^{4}$ Department of Interventional Radiology, Lishui People's Hospital of Zhejiang, \\ Lishui, Zhejiang 323000, P.R. China
}

Received October 21, 2013; Accepted April 1, 2014

DOI: $10.3892 / \mathrm{mmr} .2014 .2350$

\begin{abstract}
Subarachnoid hemorrhage (SAH), one of the serious types of stroke incurred by bleeding into the space surrounding the brain, occurs when brains are deprived of oxygen by various factors, particularly an interruption to the blood supply or a ruptured aneurysm. Cerebral vasospasm (CVS) is one of the most common complications of SAH. It has been proposed that platelet-derived growth factor (PDGF) is involved in CVS. The aim of the present study was to analyze expression of PDGF in rabbit models of CVS. Post-SAH CVS rabbit models were created using endovascular puncture and employed to analyze the expression patterns of PDGF by enzyme-linked immunosorbent assay and immunohistochemistry. The results indicated that the creation of the rabbit model of CVS induced using endovascular puncture was successful and demonstrated the double phase changes observed in human CVS. The acute stage started at $12 \mathrm{~h}$ post-SAH with narrowing of the vascular lumen diameter. This narrowing appeared again on the seventh day in delayed CVS alongside increased thickness of vessel walls. PDGF- $\beta$ expression was observed in vascular smooth muscle cells of the rabbit models. PDGF- $\beta$ was expressed as early as $3 \mathrm{~h}$ post-SAH, it was evident after 1 day and reached a peak in 7 days, suggesting that PDGF- $\beta$ is involved in the early stages of CVS. In the current study, it was confirmed that PDGF- $\beta$ expression was present in the rabbit models of CVS, which may aid the elucidation of the pathogenesis of CVS, and also provide useful information for diagnosis and treatment of CVS.
\end{abstract}

Correspondence to: Professor Rui-Min Yang, Department of Interventional Radiology, The First Affiliated Hospital of Xinxiang Medical University, 88 Health Road, Weihui, Henan 453100, P.R. China

E-mail: yangruimin893@yeah.net

Key words: subarachnoid hemorrhage, cerebral vasospasm, platelet-derived growth factor- $\beta$

\section{Introduction}

Subarachnoid hemorrhage $(\mathrm{SAH})$, one of the serious and life-threatening types of stroke incurred by bleeding into the space surrounding the brain, occurs when brains are deprived of oxygen by various factors, particularly an interruption to the blood supply or a ruptured aneurysm (1). Approximately 5-10\% of all strokes are induced by SAH resulting from ruptured berry aneurysms. There are 200,000 new SAH patients in China each year, which account for $\sim 6-8 \%$ of all stroke diagnosis (1). Due to the poor prognoses resulting from $\mathrm{SAH}$, the mortality rate of SAH patients is $\sim 50 \%$, which accounts for $22-35 \%$ of the mortalities of all cerebral vasospasm (CVS) patients. Only 20-35\% SAH patients recover their full health (1).

CVS is one of the most common complications of SAH patients. In 1959, Beard et al (2) first discovered narrowing of the arterial diameter in patients that had experienced SAH. Ecker and Riemenschnieder described clinical characteristics of CVS in detail in an analysis of cerebral angiography images in 1951. Subsequently, Ecker and Riemenschneider (4) were the first to outline the concept of CVS and described the clinical features mentioned above, which attracted the interest of the neurosurgical community. It is generally accepted that cerebral ischemia and cerebral infarction induced by CVS are the main causes of mortality and disability, and 15-30\% patients suffer from delayed ischemic neurological deficits. Although the pathogenesis of CVS has been studied for the past 50 years, the mechanism remains unclear (4).

Currently, the treatments for SAH involve prevention of vasospasm, relief of blood pressure in the brain, cessation of bleeding, and restoration of normal blood flow. SAH is mainly diagnosed using urgent computed tomography (CT) scanning without contrast, combined with radiology. However, a lumbar puncture is used following a negative CT scan. Therefore, $\sim 99 \%$ SAH can be ruled out by non-contrast CT and CT angiography in the brain (1). More effective and accurate diagnosis and treatment methods, and criteria for clinical practices are required (1).

Platelet-derived growth factor (PDGF) is a mitogenic growth factor located in platelets (5). Borel et al (6) observed that the levels of PDGF- $\beta$ increased following SAH in human 
patients, which may be associated with peripheral thrombosis, and therefore contribute to CVS. Increased levels of PDGF were also detected in ischemic brain injury and brain puncture wounds in recent studies $(5,6)$. However, the expression of PDGF in patients that experience CVS following $\mathrm{SAH}$ remains unknown.

In the present study, post-SAH CVS rat models were created using endovascular puncture and employed to analyze the expression patterns of PDGF by enzyme-linked immunosorbent assay (ELISA) and immunohistochemistry (IHC).

\section{Materials and methods}

Reagents. A PDGF- $\beta$ IHC kit was purchased from Santa Cruz Biotechnology, Inc. (Santa Cruz, CA, USA). A PDGF- $\beta$ ELISA kit was purchased from BD Biosciences (San Jose, CA, USA).

Animals. A total of 66 healthy New Zealand rabbits were provided by the Animal Center of the Medical College of Soochow University (Suzhou, China). For experiments involving animals, approval was obtained from the Institutional Review Committee of Soochow University. Animals were randomly divided into three groups: The normal control group; the sham surgery group (a guide wire was inserted into arteria carotis interna without piercing the vessel); and the SAH group. SAH subgroups were prepared at time points of 3 and $12 \mathrm{~h}$, and 1, 2, 3, 7 and 14 days following $\mathrm{SAH}$, with each subgroup containing 5 rabbits. The normal control group and the sham surgery group included 5 rabbits each. Throughout the experiment, 21 rabbits died due to the procdedure performed in the present study.

CVS rabbit models. Post-SAH CVS rabbit models were created using the endovascular puncture method. Briefly, experimental rabbits were anesthetized by a $3 \%$ pentobarbital (2.0-3.0 ml/kg) injection through veins in the ears. The rabbits were oriented in the supine position under racks with their heads and limbs fixed. The heads of the rabbits were straightened out. Rabbits were placed on DSA machine tool (innova310; General Electric, Fairfield, CT, USA) and the fur of the inguinal region was shaved. Rabbits were disinfected with iodophors and placed on aseptic towel sheets. A cut of $1.5-2.0 \mathrm{~cm}$ was made to the top of the right inguinal artery, and the arteria femoralis was separated. The catheter insertion was performed according to the method previously described in the study by Gokal et al (7). A Y valve was inserted into the upper segment of the carotid artery at the junction of the internal and external carotid arteries, by replacing the catheter guide wire using fluoroscopic surveillance.

CT examination. Unenhanced CT scanning of the rabbit heads was conducted in the various groups prior to surgery. Scanning parameters were as follows: Depth of stratum, $2.5 \mathrm{~mm}$; spacing, $2.5 \mathrm{~mm}$; field of view, $80 \mathrm{~mm}$ and 16 layers. Quantity evaluation of SAH was performed as described by the Fisher grading method (1). Unclear definition of the subarachnoid space along with a pointed-shaped high density image was designated as class I (1). Annular high density image of the subarachnoid space along with multiple levels was designated as class II (1). High density image of the subarachnoid space along with multiple levels was designated as class III (1). Unenhanced CT scanning of the rabbit heads was conducted in the different time point groups prior to execution in order to evaluate SAH changes.

Blood sample preparation. Whole blood $(5 \mathrm{ml})$ was drawn from the femoral veins of each rabbit prior to execution using a disposable syringe. Blood was rested in tubes for $30 \mathrm{~min}$, and centrifuged for $10 \mathrm{~min}$ at a speed of 5,000 x g to separate the serum and collect the supernatant. Supernatants were stored in a freezer at $-20^{\circ} \mathrm{C}$ for subsequent use.

Perfusion. The rabbits were sacrificed by intravenous injection with pentobarbital sodium. Subsequently, the chest skin of sacrificed rabbits in the different time point groups was prepared, and the pleura and pericardium were exposed with an incision $1.5 \mathrm{~cm}$ from the subcostal area between the two sides of the breastbone. When the heart and aortic root were exposed completely, an infusion needle was inserted at the root of the ascending aorta and fixed with a vascular clamp. The right atrium was cut open to drain blood. The inferior vena cava was closed using a clamp in order to exclude backflow of blood. Perfusion was conducted using $0.1 \mathrm{~mol} / 1$ phosphate-buffered saline in a $50-\mathrm{ml}$ syringe. When clear liquid effused from the right atrium, the intracranial artery and brain tissue were collected by opening the skull, and then fixed with fixative solution, including $4 \%$ paraformaldehyde and phosphate buffer.

Hematoxylin and eosin (HE) staining. The rabbits were subjected to routine pathological examinations. When slicing, cross sections and neighboring brain tissues of the superior segment of the basilar artery (BA) were selected, in addition to vascular tissues and neighboring brain tissues of the posterior communicating artery $(\mathrm{PCoA})$. Sections were prepared by paraffin wax embedding, and routine HE staining was performed. Vessel diameters and the thickness of vessel walls of the BA and PCoA were measured under HE staining and x100 magnification fields. Vessel diameters were represented as the mean of the vessel diameters of axis of bank and lateral axis within the lumen, and vessel thickness was measured by Ilker $\mathrm{G}$ method $(1,2)$.

PDGF- $\beta$ measurement. PDGF- $\beta$ levels in the sera were measured using ELISA with the PDGF- $\beta$ kit. The concentration of PDGF- $\beta$ was calculated according to the optical density values of the serum samples and the constructed standard curve.

$I H C$. To examine the expression of PDGF- $\beta$ in vascular smooth muscle cells in the present study, the streptavidin-biotin immunoperoxidase method and IHC (PDGF- $\beta$ IHC kit) were employed according to the manufacturer's instructions, and were performed as the methods elsewhere $(5,6)$. Semi-quantitative analysis was conducted according to the ratio of the positive cells and the color of the staining. The Q-test was used to compare the groups.

Diagnosis criteria for CVS. In order to define post-SAH CVS in the current study, a narrowing of the lumen diameter of the large vessels was regarded as the necessary criterion for diagnosis. 
A

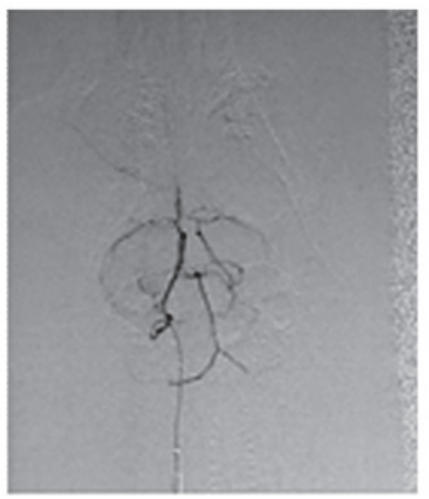

C

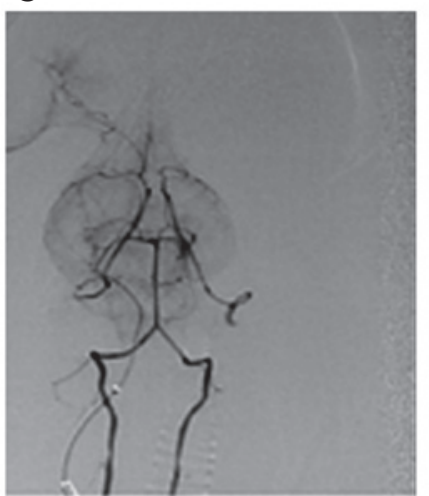

B

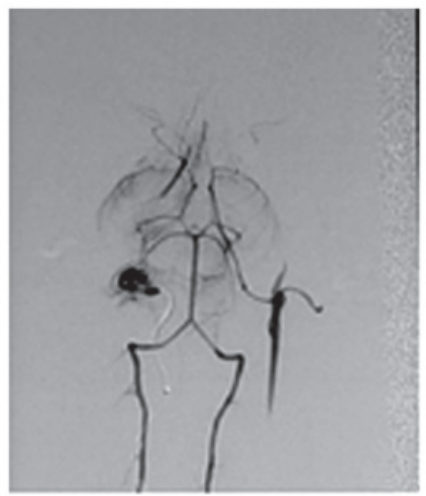

D

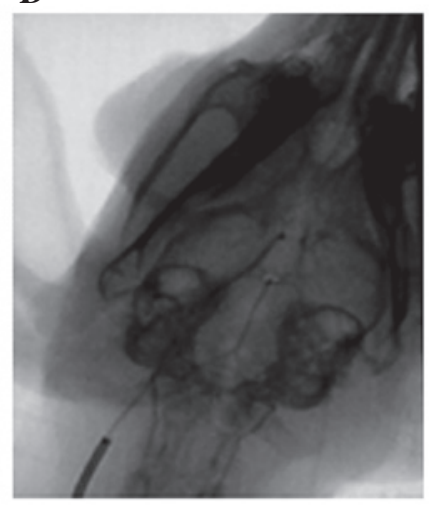

Figure 1. Representative images internal carotid artery angiography results of various rabbit groups. (A) Prior to puncture. (B) Immediate spasm following puncture. (C) Wire puncture of internal carotid artery. (D) Wire artery inserted into the internal carotid artery and then into the anterior cerebral artery.

Statistical analysis. The data are presented as the mean \pm standard deviation. The statistical significance of the data was determined by one-way analysis of variance and the F-test in SPSS version 13.0 (SPSS, Inc., Chicago, IL, USA). P<0.05 was considered to indicate a statistically significant difference. $\mathrm{P}<0.01$ was considered to indicate an highly statistically significant difference.

\section{Results}

Construction of SAH models. SAH is incurred by bleeding into the subarachnoid space of the brain, and CVS is one of the most common complications experienced by patients following SAH. To elucidate the mechanism of SAH and provide useful information for clinical practice, $\mathrm{SAH}$ rabbit models were created. A total of $35 \mathrm{SAH}$ rabbit models were successfully created out of all 66 rabbits, with a success rate of $62.5 \%$. The internal carotid artery angiography results from various groups are represented in Fig. 1.

CT scanning results. The subarachnoid space was clearly visible in CT scans of the rabbits prior to surgery (Fig. 2). Examination of the skulls of the 35 successfully constructed SAH rabbit models, and CT scans of the heads following surgery demonstrated that there were 8 cases of class I, 16 cases of class II and 11 cases of class III SAH. There was no evidence of subdural or intracranial hematoma, or changes

A

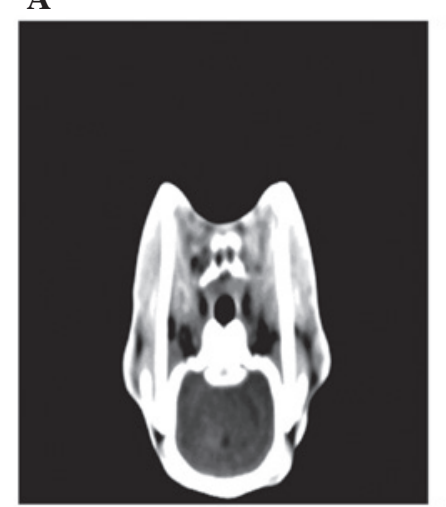

C

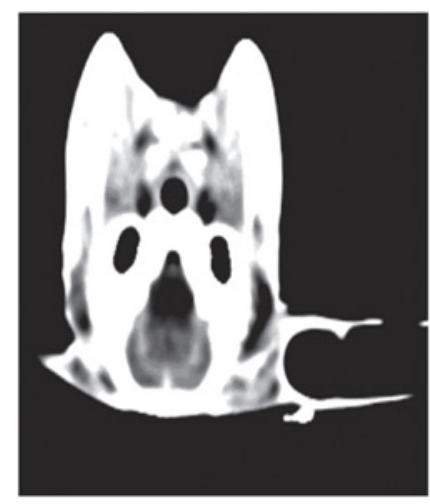

D

B
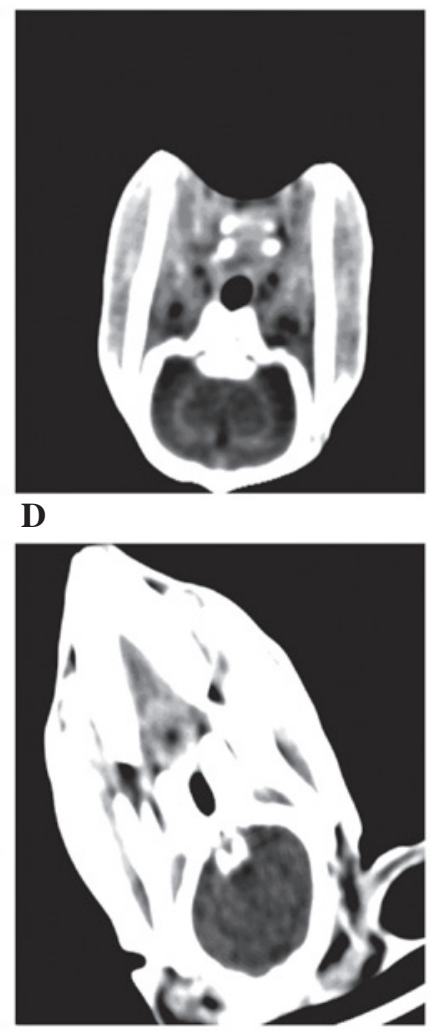

Figure 2. Representative computed tomography scans of various rabbit groups. (A) Normal control brain. (B) Spider blood II in SAH rabbits at day 3 . (C) Spider blood III in SAH rabbits at day 7. (D) SAH rabbit at day 6. SAH, subarachnoid hemorrhage.

to pneumocephalus for all the normal control, sham surgery and model rabbits. Pre-sacrifice CT scanning of rabbit heads at different time points demonstrated that there was less rabbit subarachnoid blood at the $3 \mathrm{~h}$ and 2 day time points than that in the pre-operative rabbits. There were clear subarachnoid spaces in 4 cases in the groups between 3 and 14 days. There were changes to pneumocephalus and intracranial hematoma with varying amounts of bleeding, in the rabbits with unintentional mortality. The rabbits that accidently died were scanned immediately. The animals were checked every 1-2 days.

Animal ethology. The SAH rabbit groups exhibited numerous characteristics including somnolence, weakness and anorexia in the 12-16 $\mathrm{h}$ following surgery. There were 2 rabbits that slept for 3 days, and 23 rabbits with a significant improvement in mental status evaluated by the Short Portable Mental Status Questionnaire by 1 day post-SAH compared with that immediately after SAH. The 14 day SAH group completely recovered, and there was 1 rabbit in the 3 day group with a significant improvement in mental status just $3 \mathrm{~h}$ post-SAH, comparable to the class II SAH, but it died accidently the following day.

Vasospasm morphology. The narrowing of the PCoA occurred throughout the 1-7 day subgroups, and there was a $31.2 \%$ reduction in the 7 day subgroup (this was the greatest decrease compared with the 1 day subgroup). There were significant differences between the narrowing of lumens in the SAH groups and in the normal control or sham surgery 
A

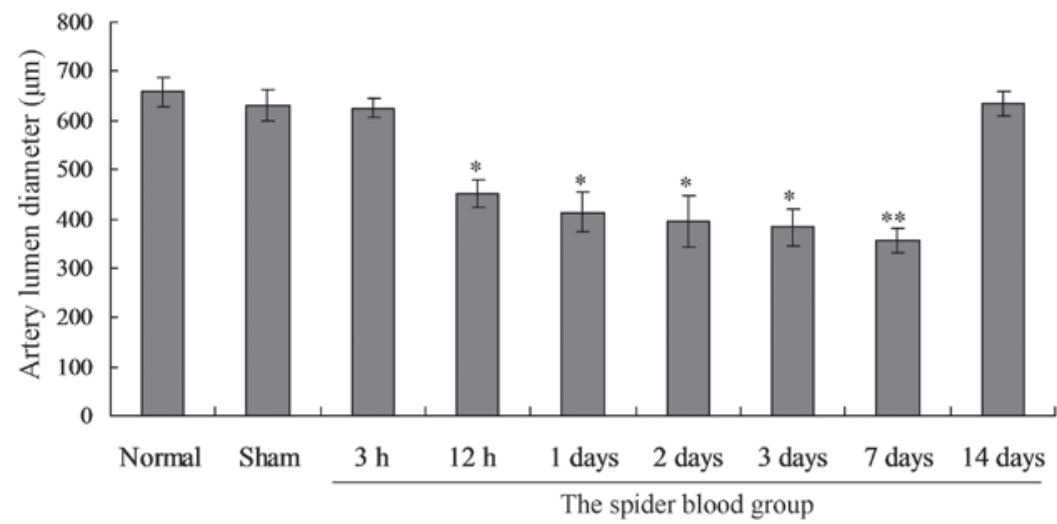

B

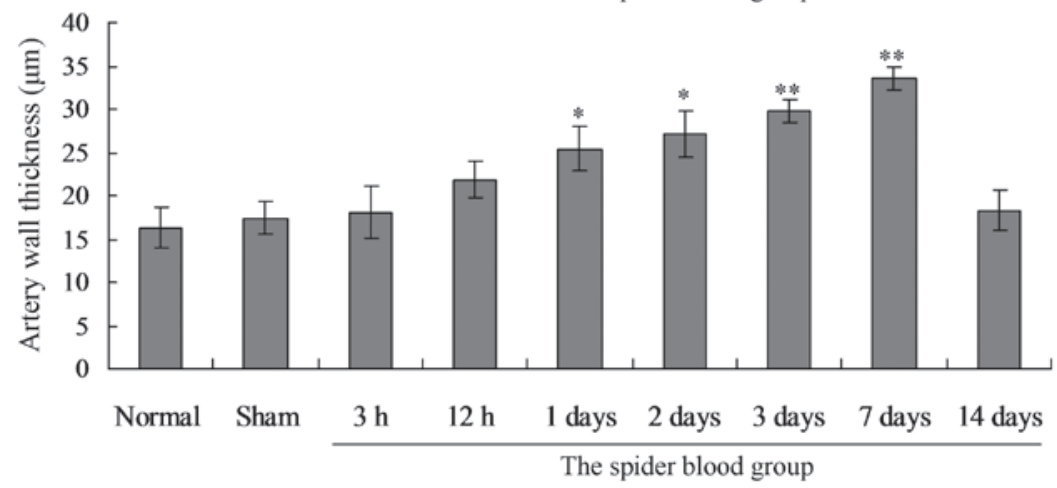

Figure 3. Posterior communicating artery. (A) Lumen diameter; and (B) wall thickness. ${ }^{*} \mathrm{P}<0.05,{ }^{* *} \mathrm{P}<0.01$ vs. the normal group.

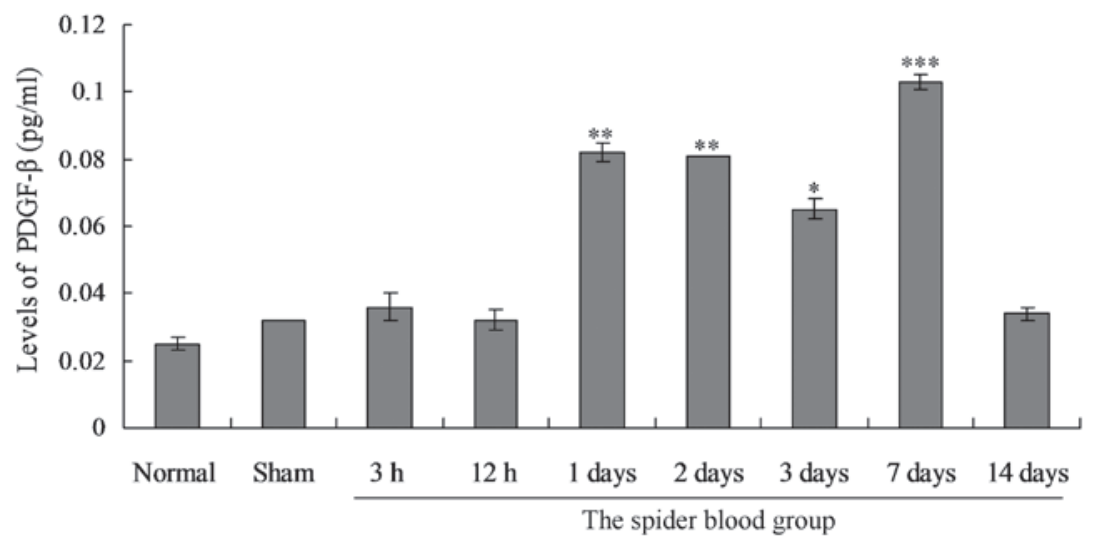

Figure 4. Expression levels of PDGF- $\beta$ in serum from the different groups. ${ }^{*} \mathrm{P}<0.05,{ }^{* *} \mathrm{P}<0.01,{ }^{* * * *} \mathrm{P}<0.001$ vs. the normal group.

groups $(\mathrm{P}<0.01)$. Statistical differences in the $3 \mathrm{~h}$ group were observed. However, there was no difference in the groups of 12 h, 1 day, 2 days, 3 days, 7 days and 14 days (Fig. 3A).

The thickness of the PCoA wall increased markedly in the 7 day group compared with that of the normal control group. Statistically significant differences in the vessel wall thickness between the 3 and $12 \mathrm{~h}$, and 1, 2 and 3 day SAH groups and the normal control or sham surgery group were observed. Specifically, there was a $58.1 \%$ increase in vessel wall thickness compared with that of the control and the sham surgery groups. No statistically significant difference was identified between other groups (Fig. 3B).

Similar to the PCoA, the diameter of the BA lumen narrowed significantly (decrease of 52.3\%) in the 7 day group compared with that in the normal control group. The thickness of the BA wall increased in the 7 day group (48.8\% increase) compared with that of the control group and the sham surgery group (data not shown).

PDGF- $\beta$ expression in serum of SAH rabbit models. The expression of PDGF- $\beta$ in serum was detected in all groups. Serum PDGF- $\beta$ expression levels were not significantly different between the normal control group and the sham surgery group ( $\mathrm{P}>0.05)$ (Fig. 4). In the SAH groups, expression of PDGF- $\beta$ was first detected in the $3 \mathrm{~h}$ group at low levels. The PDGF- $\beta$ expression levels were markedly higher in the 1 day group, and greatest in the 7 day group. Significant differences in the expression levels of PDGF- $\beta$ between the SAH groups and controls were observed. Furthermore, PDGF- $\beta$ was highly expressed in the majority of SAH groups 

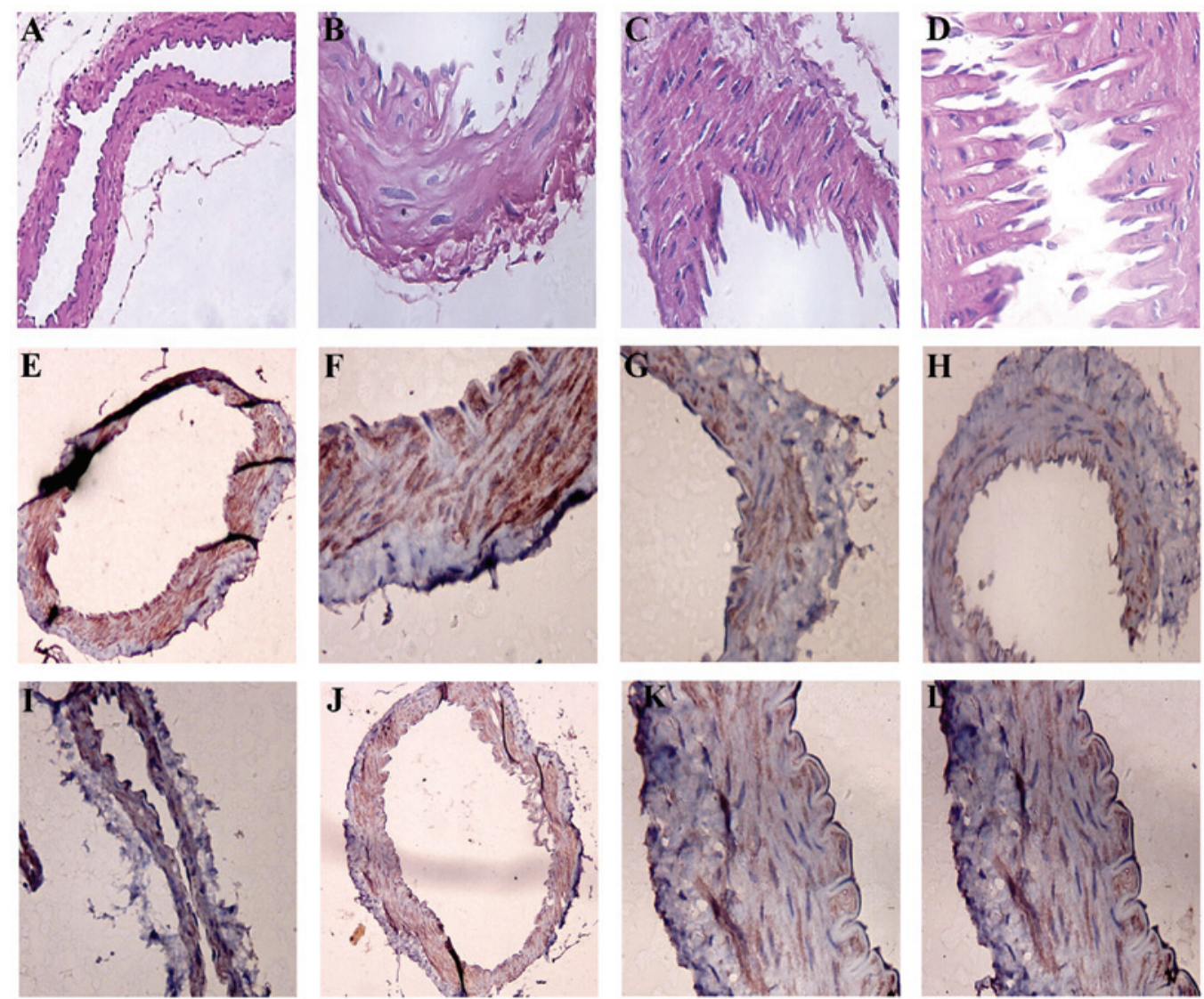

Figure 5. Results of hematoxylin and eosin (HE) staining in various groups. (A) Normal basilar artery (staining, HE; magnification, x100). (B and C) Basilar artery wall is slightly thickened in the 3 day subarachnoid hemorrhage (SAH) group (staining, HE; magnification, x400). (D) Posterior communicating artery stenosis wall thickness in the 7 day SAH group (staining HE; magnification, $\mathrm{x} 400$ ). (E) The normal traffic artery, smooth muscle cells of platelet-derived growth factor $\beta$ (PDGF- $\beta$ ) (staining, SP; magnification, $x 100$ ). (F) Normal basilar artery, smooth muscle cells of PDGF- $\beta$ (staining, SP; magnification, $x 100$ ). (G) PDGF- $\beta$ in the 3 day SAH group (staining, SP; magnification, $x 400$ ). (H) PDGF- $\beta$ in the 2 day SAH group (staining, SP; magnification, $x 400$ ). (I) PDGF- $\beta$ in the 1 day SAH group (staining, SP; magnification, $x 100$ ). (J) PDGF- $\beta$ in the 7 day SAH group (staining, SP; magnification, $x 100)$. (K) PDGF- $\beta$ in the 1 day SAH group (staining, SP; magnification, $x 400$ ). (L) PDGF- $\beta$ in the 7 day SAH group (staining, SP; magnification, $x 400$ ). SP, streptavidin peroxidase.

Table I. PDGF- $\beta$-positive cells and the gray intensity scan.

\begin{tabular}{lcc}
\hline Groups & $\begin{array}{c}\text { Gray intensity of } \\
\text { positive signals }\end{array}$ & $\begin{array}{c}\text { No. of } \\
\text { positive cells }\end{array}$ \\
\hline Normal control & $155.55 \pm 2.02$ & $33.00 \pm 4.00$ \\
Sham surgery & $156.66 \pm 2.22$ & $29.00 \pm 6.60$ \\
$3 \mathrm{~h}$ & $149.64 \pm 2.51^{\mathrm{a}}$ & $68.20 \pm 5.20^{\mathrm{b}}$ \\
$12 \mathrm{~h}$ & $151.90 \pm 1.88^{\mathrm{a}}$ & $64.20 \pm 6.72^{\mathrm{b}}$ \\
1 day & $142.99 \pm 3.27^{\mathrm{b}}$ & $89.00 \pm 5.20^{\mathrm{b}}$ \\
2 day & $150.88 \pm 1.88^{\mathrm{a}}$ & $74.60 \pm 9.80^{\mathrm{b}}$ \\
3 day & $146.50 \pm 2.20^{\mathrm{a}}$ & $80.80 \pm 7.70^{\mathrm{b}}$ \\
7 day & $140.81 \pm 3.34^{\mathrm{b}}$ & $110.60 \pm 9.24^{\mathrm{b}}$ \\
14 day & $153.05 \pm 1.97^{\mathrm{a}}$ & $66.40 \pm 6.77^{\mathrm{b}}$ \\
\hline
\end{tabular}

${ }^{\mathrm{a}} \mathrm{P}<0.05$ and ${ }^{\mathrm{b}} \mathrm{P}<0.01$ vs. normal group.

throughout the experiment $(\mathrm{P}<0.05)$, and the levels of PDGF- $\beta$ in the 14 day group were higher than those of the control and sham surgery groups.

Histopathology results. In the control and sham surgery groups, the structure of the vessel walls was normal, and the tunica intima consisted of endothelial cells with normal structures (Fig. 5). The tunica media consisted of smooth muscle cells and their surrounding extracellular matrices. The tunica adventitia was composed of fibroblasts and loose connective tissues. In comparison, all vessel endothelial cells were disrupted in all SAH groups; intercellular spaces of smooth muscle cells increased in size, with changes to the cytoplasm, vacuoles and nuclear membrane; and a condensed nucleus were present in a number of cells. One day following $\mathrm{SAH}$, the nuclei of the endothelial cells changed (with the condensed nucleus) and this was most evident in the 2 and 3 day groups. There were also aggregated platelets in the endothelial cells, which was most clear in the 7 day group, and elastic membranes were twisted.

Histopathology of PDGF- $\beta$. In the control group and the sham surgery group, the nuclei of smooth muscle cells were blue and there was no expression of PDGF- $\beta$ (Fig. 5). In PDGF- $\beta$-positive cells, the cytoplasm and cytomembranes were pale brown, and the nuclei were not stained (Fig. 5). A small number of PDGF- $\beta$-positive cells from the PCoA were present in the 3 and $12 \mathrm{~h}$ SAH groups. There were PDGF- $\beta$-positive smooth muscle cells in the 1 day group, which were observed in the 2 and 3 day groups and peaked in 7 days (Table I). By contrast, 
the 14 day group had markedly lower numbers of positive cells in comparison to the days 1-7 groups, which were still slightly higher than the number in the normal control and the sham surgery groups. With respect to PDGF- $\beta$ expression levels, statistically significant differences between the 1,2 and 3 day groups and the normal control and sham surgery groups were observed $(\mathrm{P}<0.05)$. There were statistically significant differences between the 1 and 2 day groups, and the $12 \mathrm{~h}$ and 1 day groups with respect to PDGF- $\beta$ expression levels $(\mathrm{P}<0.05)$.

\section{Discussion}

Thus far, the mechanisms of the pathogenesis of CVS are unknown, so it is necessary to create novel, more reliable animal models to further investigate these mechanisms. Megyesi et al (8) reported that $28 \%$ of CVS animal models were created using endovascular puncture or vascular laceration, and $72 \%$ by injecting blood into the subarachnoid space or inserting a blood clot in vessels to induce convulsion. However, there are various limitations to these SAH animal models. For example, there is a high mortality rate in mouse SAH models, so the models are mainly used in pathological and physiological analysis of acute CVS. Furthermore, there are numerous differences in vascular anatomy between mice and humans. Cerebral ischemia and spasm do not usually appear throughout the rich collateral circulation in such a short time window $(8,9)$. Therefore, a novel animal model for $\mathrm{SAH}$ is required. Rabbit SAH models were first reported by Offerhau and Van Gool (7) in 1969. The periods of time prior to spasm in the models were consistent with those in humans, so they have been widely employed in SAH and CVS studies. In the present study, rabbit CVS models were constructed and used. The mortality rate of the model was $\sim 37.2 \%$, which is consistent with of previous studies (7,10-12). The limitations of the rabbit model in the present study included a high mortality rate and high levels of intracranial bleeding, which may be alleviated by improvements to surgical methods and instruments.

Gules et al (13) discovered that the establishment method of SAH model was not effective. There were also five rabbits that did not establish the SAH model. Therefore, our result was consistent with the study by Gules et al (13). The reason may be that the micro-wire hard-head did not enter intracranial artery due to the tortuosity of the initial part of the intracranial vessels. As the effects of bleeding on the pathological and physiological changes of vessels were difficult to evaluate in the CVS rabbit models induced by endovascular puncture in the present study, CT scans of heads were used and changes were evaluated according to Fisher rating methods. Survival rates were high in class I and II cases, with clear changes in vessel pathology. Additionally, spider blood disappeared quickly, mostly in 2-3 days, and there was no spider blood in the 14 day group based on the CT scan analysis. It was reported by Woodcock et al (11) that magnetic resonance imaging fluid-attenuated inversion recovery was better and more sensitive than CT in diagnosing acute subarachnoid, which is consistent with the present study.

PDGF is an important mitogenic agent that enhances cell proliferation of vascular smooth muscle cells, and there is a positive parallel correlation between expression of PDGF in the injured vascular smooth muscle cells and cell proliferation (14). Roles of PDGF in the occurrence and development of CVS have attracted the interest of numerous researchers since Borel et al (6) reported that PDGF- $\beta$ expression levels were greater in SAH patients compared with those of controls, and that PDGF was involved in cell proliferation in mouse convulsion models. Increased expression levels of PDGF were also detected in ischemic brain injury and brain puncture wounds in a previous study. Vieweg et al (12) found that levels of PDGF- $\beta$ in serum of dog convulsion models increased in the third day and the seventh day, which was not correlated with convulsion in radiography analysis. Although PDGF is expressed in vascular endothelial cells and smooth muscle cells in human patients and animal models, there has been little study of the expression of PDGF in vasospasm animal models induced by vascular rupture hemorrhage similar to that of humans. Therefore, expression patterns of PDGF- $\beta$ in post-SAH CVS rabbit models induced by endovascular puncture method were analyzed in the present study. Specifically, levels of serum PDGF- $\beta$ were analyzed using ELISA and expression levels of PDGF- $\beta$ in vascular smooth muscle cells were examined by IHC.

The results demonstrated that PDGF- $\beta$ was expressed in the $3 \mathrm{~h}$ post-SAH group, but not in the $12 \mathrm{~h}$ group. The expression levels of PDGF- $\beta$ increased in the 1 day group, and the expression was clear in the 2 and 3 day groups. In addition, the levels of PDGF- $\beta$ peaked in the 7 day group, and expression levels of PDGF- $\beta$ decreased in the 14 day group, but were still higher than those of the normal control group and the sham surgery group. The majority of PDGF- $\beta$-positive cells were smooth muscle cells at the earlier stages, the number of which increased in the 1 day group and peaked in 7 day group. There were PDGF- $\beta$-positive endothelial cells in some small vessels. Time-course expression of PDGF- $\beta$ was consistent with the activation and proliferation of smooth muscle cells. There were PDGF- $\beta$-positive endothelial cells and vascular smooth muscle cells, indicating that PDGF- $\beta$ may participate in activation and proliferation of smooth muscle cells, and pathogenesis and development of vascular proliferation. This is consistent with the findings of Borel et al (6), who also observed that PDGF- $\beta$ was highly expressed 7 days subsequent to $\mathrm{SAH}$.

Human chronic CVS occurs $\sim 3$ days following $\mathrm{SAH}$, peaks at 6-7 days, and then is alleviated by $\sim 14$ days (15). In the present study, there was further narrowing in the 7 day group which was alleviated in the 14 day group, consistent with patterns in humans. Vessel wall thickness in the BA and PCoA were greatest in the 7 day group than that at every other time point, which is consistent with the high expression levels of PDGF- $\beta$ in the 7 day group. This may be due to smooth muscle cell spacing and numbers increased, and collencyte and fibroblast numbers increased, leading to an enhanced thickness of the vessel wall and narrowed lumen. However, the underlying mechanism remains to be investigated. Future studies should focus on time-course expression of PDGF- $\beta$ in animal models and clinical samples along with large-sample statistics. Occurrence, development and the signaling pathways in CVS should also be emphasized.

In conclusion, there was PDGF- $\beta$ expression in the CVS rabbit models in the present study, which may aid the 
elucidation of the pathogenesis of CVS, and also provide useful information for diagnosis and treatment of CVS.

\section{Acknowledgements}

This study was supported by a grant of a Research Contact with the Science and Technology Agency of Henan Province (no. 082102310014).

\section{References}

1. Lee CI, Chou AK, Lin CC, et al: Immune and inflammatory gene signature in rat cerebrum in subarachnoid hemorrhage with microarray analysis. Mol Med Rep 5: 118-125, 2012.

2. Beard EF, Robertson JW and Robertson RC: Spontaneous subarachnoid hemorrhage simulating acute myocardial infarction. Am Heart J 58: 755-759, 1959.

3. Ecker A and Riemenschnieder PA: Arteriographic demonstration of spasm of the intracranial arteries, with special reference to saccular arterial aneurysms. J Neurosurg 8: 660-667, 1951.

4. Ecker A and Riemenschneider PA: Arteriographic evidence of spasm in cerebral vascular disorders. Neurology 3: 495-502, 1953.

5. Rosiak M, Postula M, Kaplon-Cieslicka A, et al: Lack of effect of common single nucleotide polymorphisms in leukotriene pathway genes on platelet reactivity in patients with diabetes. Mol Med Rep 8: 853-860, 2013.

6. Borel CO, McKee A, Parra A, et al: Possible role for vascular cell proliferation in cerebral vasospasm after subarachnoid hemorrhage. Stroke 34: 427-433, 2003.
7. Gokal R, Alexander S, Ash S, Chen TW, Danielson A and Holmes C: Peritoneal catheters and exit-site practices towards optimum peritoneal access: 1998 update. Perit Dial Int 18: 11-33, 1998.

8. Offerhaus L and van Gool J: Electrocardiographic changes and tissue catecholamines in experimental subarachnoid haemorrhage. Cardiovasc Res 3: 433-440, 1969.

9. Shaw MD, Vermeulen M, Murray GD, et al: Efficacy and safety of the endothelin, receptor antagonist TAK-044 in treating subarachnoid hemorrhage: a report by the Steering Committee on behalf of the UK/Netherlands/Eire TAK-044 Subarachnoid Haemorrhage Study Group. J Neurosurg 93: 992-997, 2000.

10. Mayberg MR, Okada T and Bark DH: The significance of morphological changes in cerebral arteries after subarachnoid hemorrhage. J Neurosurg 72: 626-633, 1990.

11. Woodcock RJ Jr, Short J, Do HM, et al: Imaging of acute subarachnoid hemorrhage with a fluid-attenuated inversion recovery sequence in an animal model: comparison with non-contrast-enhanced CT. AJNR Am J Neuroradiol 22: 1698-1703, 2001

12. Vieweg U, Schramm J and Urbach H: Platelet-derived growth factor (PDGF-AB) like immune reactivity in serum and in cerebral spinal fluid following experimental subarachnoid haemorrhage in dogs. Acta Neurochir (Wien) 141: 861-866, 1999.

13. GulesI,Satoh M,Clower BR, et al: Comparison of three rat models of cerebral vasospasm. Am J Physiol Heart Circ Physiol 283: $\mathrm{H} 2551-\mathrm{H} 2559,2002$.

14. Ellis EF, Nies AS and Oates JA: Cerebral arterial smooth muscle contraction by thromboxane A2. Stroke 8: 480-483, 1977.

15. Hou HW, Li XG, Yan M, et al: Increased leukocyte Rho-kinase activity in a population with acute coronary syndrome. Mol Med Rep 8: 250-254, 2013. 